\title{
Insular cortex is involved in consolidation of object recognition memory
}

\author{
Federico Bermudez-Rattoni, ${ }^{1,2,4}$ Shoki Okuda, ${ }^{1,3}$ Benno Roozendaal, ${ }^{1}$ \\ and James L. McGaugh' \\ ${ }^{1}$ Center for the Neurobiology of Learning and Memory and Department of Neurobiology and Behavior, University of California, \\ Irvine, California 92697-3800, USA; ${ }^{2}$ Departamento de Neurociencias, Instituto de Fisiología Celular, Universidad Nacional \\ Autónoma de México, México D.F., 04510, México; ${ }^{3}$ CNS Disorder Research, Tsukuba Research Institute, Banyu Pharmaceutical \\ Co., Tsukuba, Ibaraki, 300-2611, Japan
}

Previous findings indicate that the insular cortex (IC) is involved in conditioned taste aversion and taste recognition. Here we report evidence that the IC is not uniquely dedicated to taste learning but plays a more general role in consolidating recognition memories. Immediate post-training infusions of the muscarinic cholinergic receptor antagonist scopolamine administered into the IC produced locus-specific and time-dependent impairment of object recognition memory.

Extensive evidence indicates that the IC, also termed gustatory cortex, is critically involved in conditioned taste aversion and taste recognition memory (Bermudez-Rattoni 2004). Although most studies of the involvement of the IC in memory have investigated taste, there is some evidence that the IC is involved in memory that is not based on taste (Bermudez-Rattoni and McGaugh 1991; Bermudez-Rattoni et al. 1991; Paller et al. 2003; Reed et al. 2004). Converging evidence from animal and human studies suggests that a network of temporal cortical regions participates in recognition memory (Tang et al. 1997; Aggleton and Brown 1999; Malkova and Mishkin 2003; Reed et al. 2004). However, there is little information on the role of the IC in visual recognition memory. Therefore, the present study examined the involvement of the IC in the consolidation of object recognition memory, a task based on the tendency of rodents to explore a novel object more than a familiar one (Ennaceur and Delacour 1988). As microdialysis studies have shown that the release of acetylcholine in the IC in response to a novel, but not a familiar, taste stimulus (Miranda et al. 2000) and cortical cholinergic activity is involved in the acquisition of object recognition memory (Tang et al. 1997; Warburton et al. 2003), the present study examined whether the muscarinic cholinergic receptor antagonist scopolamine infused into the IC immediately after training impairs the consolidation of object recognition memory. To control for site specificity, other rats received posttraining infusions of scopolamine into the frontoparietal cortex (FPC), dorsomedial but adjacent to the IC. All experimental procedures were in compliance with NIH guidelines and approved by the UC Irvine's Institutional Animal Care and Use Committee.

Adult male Sprague-Dawley rats (280-320 g at time of surgery) from Charles River Laboratories were implanted under sodium pentobarbital anesthesia $(50 \mathrm{mg} / \mathrm{kg}$, ip) with bilateral guide cannulae (23-ga.) aimed either at the IC [coordinates of the infusion sites from bregma (Paxinos and Watson 1986): anteroposterior (AP), $+1.2 \mathrm{~mm}$; mediolateral $(\mathrm{ML}), \pm 5.5 \mathrm{~mm}$; dorsoventral (DV), $-6.5 \mathrm{~mm}$ ] or the FPC (AP, $+1.2 \mathrm{~mm}$; ML, $\pm 4.0 \mathrm{~mm}$; DV, $-3.0 \mathrm{~mm}$ ). Histological examination revealed that the injection

\footnotetext{
${ }^{4}$ Corresponding author.

E-mail fbermude@ifc.unam.mx; fax (5255) 56225607.

Article published online ahead of print. Article and publication date are at http://www.learnmem.org/cgi/doi/10.1101/lm.97605.
}

needle tips were placed in the granular and agranular divisions of the IC, from -0.3 to $+1.7 \mathrm{~mm}$ from bregma (Fig. 1A). Needles aimed at the FPC were found in the dorsal FPC from -0.8 to +1.7 $\mathrm{mm}$ from bregma (Fig. 1B). Three animals with cannulae misplacements were excluded from analyses, leaving 8-11 animals per group.

After recovery, the rats were trained and tested on an object recognition task (for general procedures, see Okuda et al. 2004). Rats were initially habituated to the open-field box $(40 \mathrm{~cm}$ wide $\times 40 \mathrm{~cm}$ deep $\times 40 \mathrm{~cm}$ high), without any objects, for $3 \mathrm{~min}$ each on five consecutive days. On the training trial, the rat was placed in the open field for $10 \mathrm{~min}$ and allowed to freely explore two identical objects [either two opaque glass light bulbs $(6-\mathrm{cm}$ dia, $11-\mathrm{cm}$ length) or two transparent glass vials $(5.5-\mathrm{cm}$ dia, 5 -cm height)]. The time spent exploring each object and the total time spent exploring both objects were recorded. Exploration of an object was defined as pointing the nose to the object at a distance of $<1 \mathrm{~cm}$ and/or touching it with the nose. Turning around, climbing, or sitting on an object was not considered exploration. The muscarinic cholinergic receptor antagonist scopolamine (30 $\mu \mathrm{g}$ in $0.5 \mu \mathrm{L}$ of saline, Sigma) or saline was infused bilaterally (for $60 \mathrm{sec}$ ) into the IC or the FPC immediately (Sal or Sc-0) or $160 \mathrm{~min}$ (Sc-160) after the training trial. Nonoperated rats (Non-Surg) served as controls and were trained using the same procedure.

During the training trial, the groups exhibited comparable time exploring each of the two identical objects (one-sample $t$ tests: $P \geq 0.23$ ). A discrimination index was calculated as the difference in time spent exploring the two objects, expressed as the ratio of the total time spent exploring both objects (Ennaceur and Delacour 1988). One-way ANOVAs indicated no differences in the discrimination index among groups on the training trial (IC: $F_{3,33}=0.93, P=0.44 ;$ FPC: $F_{3,36}=0.54, P=0.66$; data not shown). One-way ANOVAs for total exploration time of the two identical objects on the training trial also revealed no differences between groups prior to drug treatment (Table 1).

For 24-h retention testing, the rat was placed in the experimental apparatus for $3 \mathrm{~min}$, and the time spent exploring a copy of the object used on the training trial (familiar object) and a new object was recorded. All combinations and locations of objects were used in a balanced manner to reduce potential biases due to preference for particular locations or objects. On the retention test, the discrimination index of the nonoperated rats and of those given saline into the IC were significantly different from zero (both, $P<0.0001$ ), indicating that they readily discriminated the novel object (Fig. 1C). However, rats given immediate post-training infusions of scopolamine (Sc-0) into the IC failed to show a significant exploration preference for the novel object. Delayed infusions of scopolamine into the IC (Sc-160) did not 
A

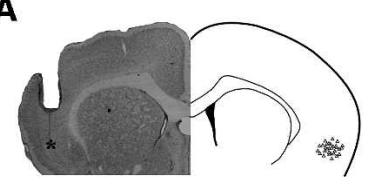

B

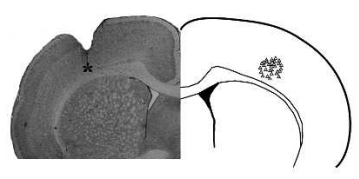

C

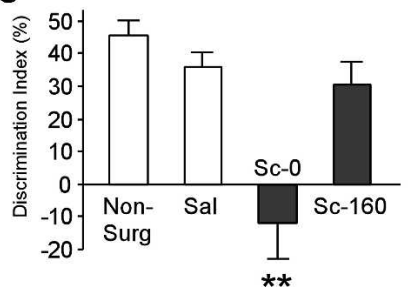

D

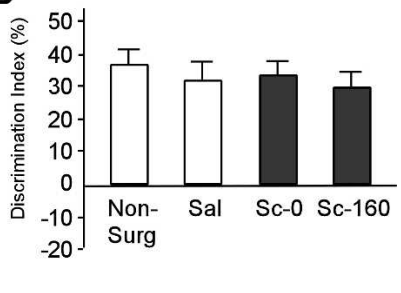

Figure 1. Scopolamine infused into the IC but not the FPC impaired the consolidation of object recognition memory. $(A, B)$ Diagram and photomicrograph illustrating placements of cannulae and needle tip in the IC $(A)$ and FPC $(B)$. (C,D) Effects of scopolamine on object recognition memory. Scopolamine $(30 \mu \mathrm{g}$ in $0.5 \mu \mathrm{L})$ or saline was infused bilaterally into the IC $(C)$ or the FPC (D) immediately (Sal and Sc-0) or $160 \mathrm{~min}$ (Sc-160) after the training trial. Data are presented as discrimination index (mean \pm SEM; see main text). ${ }^{* *}, P<0.0001$ vs. Saline group ( $n=11$ per group).

impair retention, indicating a time-limited involvement of the IC in the consolidation of object recognition memory. One-way ANOVA for discrimination index also showed a significant group effect $\left(F_{3,33}=13.54, P<0.0001\right)$, and post hoc analysis revealed that the Sc-0 group had a significantly decreased discrimination index compared to rats treated with saline (Fisher's: $P<0.0001$ ) as well as those given delayed infusions of scopolamine (Fisher's: $P<0.0005$, Fig. 1C). In contrast, neither immediate nor delayed infusions of scopolamine into the FPC impaired retention performance. Furthermore, one-way ANOVA for discrimination index for the FPC groups indicated no group differences $\left(F_{3,36}=0.35, P=0.78\right.$; Fig. 1D). In both the IC and FPC studies, one-way ANOVAs for total exploration time of the two objects on the test trial revealed no differences between groups (Table 1).

The novel finding of this study is that immediate posttraining infusions of the muscarinic cholinergic receptor antagonist scopolamine administered into the IC impaired object recognition memory, whereas scopolamine infused into the adjacent FPC did not impair memory. These findings provide compelling evidence that the IC is not engaged uniquely in the (associative) learning and recognition of taste, but that it plays a more general role in recognition memory. Such findings fit well with recent findings of two brain imaging studies suggesting an involvement of the IC in human face and tactile recognition (Paller et al. 2003; Reed et al. 2004), as well as with evidence that reversible or permanent lesions of the IC produce strong impairments in the consolidation of inhibitory avoidance and watermaze spatial learning (Bermudez-Rattoni and McGaugh 1991; Bermudez-Rattoni et al. 1991; Nerad et al. 1996).

It is well established that a network of temporal structures including the perirhinal, parahippocampal, and entorhinal cortices and the hippocampus participates in recognition memory. Our results suggest that the IC is part of this network, as it is highly involved in processing the consolidation of newly presented tastes and, as the present findings indicate, of objects. There are extensive reciprocal connections between the IC and all of these other brain regions (Swanson and Kohler 1986; McIntyre et al. 1996; Lavenex et al. 2002), suggesting a high degree of functional overlap between these different brain regions. However, particular regions of the cortical mantle appear to be more importantly involved in object recognition memory. It has been reported that transient inactivation of the perirhinal cortex and the medial prefrontal cortex produces differential effects on object recognition memory and temporal order discrimination. Whereas inactivation of the perirhinal cortex blocks the encoding of object recognition memory, similar inactivation of the medial prefrontal cortex blocks memory of recency, but not familiarity of the objects (Hannesson et al. 2004). Although more research is necessary to determine which brain areas are involved in novelty-familiarity recognition or other aspects of recognition memory, it seems that temporal cortical structures located along the rhinal sulcus, including the IC, are highly involved in recognition memory (Aggleton and Brown 1999).

Previous studies have reported that pretraining microinjections of scopolamine into monkey perirhinal cortex impair acquisition on a visual recognition memory task (Tang et al. 1997). Furthermore, scopolamine administered to rats either systemically or directly into the perirhinal cortex before training was found to disrupt acquisition and/or consolidation of recognition memory (Warburton et al. 2003). The present study, however, is the first to demonstrate that immediate post-training, but not delayed, microinfusions of scopolamine into the cortex impair object recognition memory. These findings indicate a timelimited involvement of cholinergic activation in the IC in the consolidation of recognition memory and are congruent with extensive evidence indicating a critical role of the cholinergic system of the IC in taste memory consolidation (Naor and Dudai 1996; Gutierrez et al. 2003a,b). Microdialysis studies have shown that exposure to novel environments or novel tastes produces a significant cortical release of acetylcholine, but not of glutamate or GABA (Miranda et al. 2000, 2002; Giovannini et al. 2001). However, after several presentations of a given taste or exposures to the same environment, there is a significant weakening of the acetylcholine response until it reaches baseline levels (Acquas et al. 1996; Pepeu and Blandina 1998; Miranda et al. 2000). These findings suggesting an inverse relationship between familiarity and cortical acetylcholine release fit well with the present findings indicating that muscarinic receptor blockade impairs recognition memory by preventing novel objects or tastes from becoming familiar.

In summary, our findings suggest that the IC is involved in object recognition memory and that cholinergic activity within the IC is critically involved in the consolidation of object familiarity underlying recognition memory. These results provide compelling evidence that the IC is involved in declarative memory, and they open new venues for investigating the involvement of temporal structures and molecular mechanisms in the consolidation of different kinds of declarative memory.

Table 1. Total object exploration time on the training and test trials

\begin{tabular}{lcclcc}
\hline & \multicolumn{2}{c}{ Insular cortex } & & \multicolumn{2}{c}{ Fronto-parietal cortex } \\
\cline { 2 - 3 } \cline { 6 - 6 } \cline { 6 - 7 } & Training & Test & & Training & Test \\
\hline Non-Surg & $56.8 \pm 5.0$ & $23.0 \pm 5.6$ & & $54.1 \pm 3.5$ & $14.9 \pm 1.3$ \\
Sal & $58.6 \pm 2.8$ & $17.7 \pm 1.5$ & & $50.3 \pm 6.7$ & $17.0 \pm 2.0$ \\
Sc-0 & $68.6 \pm 6.0$ & $16.1 \pm 1.8$ & & $64.4 \pm 9.7$ & $22.5 \pm 6.6$ \\
Sc-160 & $61.1 \pm 5.1$ & $22.3 \pm 2.0$ & & $59.7 \pm 4.7$ & $16.8 \pm 1.7$ \\
P-values & 0.32 & 0.33 & & 0.44 & 0.43 \\
\hline
\end{tabular}

Total time spent exploring the two objects (two identical objects for the training trial, and a familiar and a novel object for the test trial), expressed as mean \pm SEM, in sec. Scopolamine or saline was infused immediately (Sal and Sc-0) or 160 min (Sc-160) after the training trial. One-way ANOVA for total object exploration time revealed no differences between drug groups on the training and test trials. 


\section{Acknowledgments}

This research was supported by MH12526 (J.L.M.) and CONACYT 42657-Q and DGAPA.-UNAM IN-202504 (F.B.-R.).

\section{References}

Acquas, E., Wilson, C., and Fibiger, H.C. 1996. Conditioned and unconditioned stimuli increase frontal cortical and hippocampal acetylcholine release: Effects of novelty, habituation, and fear. J. Neurosci. 16: 3089-3096.

Aggleton, J.P. and Brown, M.W. 1999. Episodic memory, amnesia, and the hippocampal-anterior thalamic axis. Behav. Brain Sci. 22: 425-444; 444-489.

Bermudez-Rattoni, F. 2004. Molecular mechanisms of taste-recognition memory. Nat. Rev. Neurosci. 5: 209-217.

Bermudez-Rattoni, F. and McGaugh, J.L. 1991. Insular cortex and amygdala lesions differentially affect acquisition on inhibitory avoidance and conditioned taste aversion. Brain Res. 549: 165-170.

Bermudez-Rattoni, F., Introini-Collison, I.B., and McGaugh, J.L. 1991. Reversible inactivation of the insular cortex by tetrodotoxin produces retrograde and anterograde amnesia for inhibitory avoidance and spatial learning. Proc. Natl. Acad. Sci. 88: 5379-5382.

Ennaceur, A. and Delacour, J. 1988. A new one-trial test for neurobiological studies of memory in rats. 1: Behavioral data. Behav. Brain Res. 31: 47-59.

Giovannini, M.G., Rakovska, A., Benton, R.S., Pazzagli, M., Bianchi, L., and Pepeu, G. 2001. Effects of novelty and habituation on acetylcholine, GABA, and glutamate release from the frontal cortex and hippocampus of freely moving rats. Neuroscience 106: 43-53.

Gutierrez, R., Rodriguez-Ortiz, C.J., De La Cruz, V., Nunez-Jaramillo, L., and Bermudez-Rattoni, F. 2003a. Cholinergic dependence of taste memory formation: Evidence of two distinct processes. Neurobiol. Learn. Mem. 80: 323-331.

Gutierrez, R., Tellez, L.A., and Bermudez-Rattoni, F. 2003b. Blockade of cortical muscarinic but not NMDA receptors prevents a novel taste from becoming familiar. Eur. J. Neurosci. 17: 1556-1562.

Hannesson, D.K., Vacca, G., Howland, J.G., and Phillips, A.G. 2004. Medial prefrontal cortex is involved in spatial temporal order memory but not spatial recognition memory in tests relying on spontaneous exploration in rats. Behav. Brain Res. 153: 273-285.

Lavenex, P., Suzuki, W.A., and Amaral, D.G. 2002. Perirhinal and parahippocampal cortices of the macaque monkey: Projections to the neocortex. J. Comp. Neurol. 447: 394-420.
Malkova, L. and Mishkin, M. 2003. One-trial memory for object-place associations after separate lesions of hippocampus and posterior parahippocampal region in the monkey. J. Neurosci. 23: 1956-1965.

McIntyre, D.C., Kelly, M.E., and Staines, W.A. 1996. Efferent projections of the anterior perirhinal cortex in the rat. J. Comp. Neurol. 369: 302-318.

Miranda, M.I., Ramirez-Lugo, L., and Bermudez-Rattoni, F. 2000. Cortical cholinergic activity is related to the novelty of the stimulus. Brain Res. 882: 230-235.

Miranda, M.I., Ferreira, G., Ramirez-Lugo, L., and Bermudez-Rattoni, F. 2002. Glutamatergic activity in the amygdala signals visceral input during taste memory formation. Proc. Natl. Acad. Sci. 99: 1141711422 .

Naor, C. and Dudai, Y. 1996. Transient impairment of cholinergic function in the rat insular cortex disrupts the encoding of taste in conditioned taste aversion. Behav. Brain Res. 79: 61-67.

Nerad, L., Ramirez-Amaya, V., Ormsby, C.E., and Bermudez-Rattoni, F. 1996. Differential effects of anterior and posterior insular cortex lesions on the acquisition of conditioned taste aversion and spatial learning. Neurobiol. Learn. Mem. 66: 44-50.

Okuda, S., Roozendaal, B., and McGaugh, J.L. 2004. Glucocorticoid effects on object recognition memory require training-associated emotional arousal. Proc. Natl. Acad. Sci. 101: 853-858.

Paller, K.A., Ranganath, C., Gonsalves, B., LaBar, K.S., Parrish, T.B., Gitelman, D.R., Mesulam, M.M., and Reber, P.J. 2003. Neural correlates of person recognition. Learn Mem. 10: 253-260.

Paxinos, G. and Watson, C. 1986. The rat brain in stereotaxic coordinates, 3rd ed. Academic Press, San Diego.

Pepeu, G. and Blandina, P. 1998. The acetylcholine, GABA, glutamate triangle in the rat forebrain. J. Physiol. Paris 92: 351-355.

Reed, C.L., Shoham, S., and Halgren, E. 2004. Neural substrates of tactile object recognition: An fMRI study. Hum. Brain Mapp. 21: 236-246.

Swanson, L.W. and Kohler, C. 1986. Anatomical evidence for direct projections from the entorhinal area to the entire cortical mantle in the rat. J. Neurosci. 6: 3010-3023.

Tang, Y., Mishkin, M., and Aigner, T.G. 1997. Effects of muscarinic blockade in perirhinal cortex during visual recognition. Proc. Natl. Acad. Sci. 94: 12667-12669.

Warburton, E.C., Koder, T., Cho, K., Massey, P.V., Duguid, G., Barker, G.R., Aggleton, J.P., Bashir, Z.I., and Brown, M.W. 2003. Cholinergic neurotransmission is essential for perirhinal cortical plasticity and recognition memory. Neuron 38: 987-996.

Received May 5, 2005; accepted in revised form July 5, 2005. 


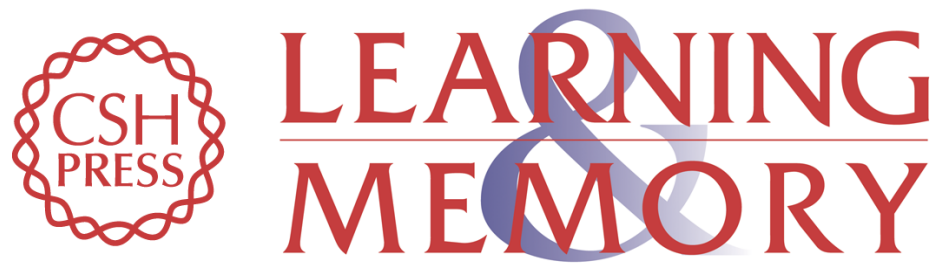

\section{Insular cortex is involved in consolidation of object recognition memory}

Federico Bermudez-Rattoni, Shoki Okuda, Benno Roozendaal, et al.

Learn. Mem. 2005, 12:

Access the most recent version at doi:10.1101/lm.97605

References This article cites 24 articles, 8 of which can be accessed free at: http://learnmem.cshlp.org/content/12/5/447.full.html\#ref-list-1

License

Email Alerting

Receive free email alerts when new articles cite this article - sign up in the box at the Service top right corner of the article or click here. 